\title{
CS(3-2) in Nearby Starburst Galaxies M82 and NGC253
}

\author{
Sumio ISHIZUKI ${ }^{1}$, Ryohei KAWABE, and Masato ISHIGURO \\ Nobeyama Radio Observatory
}

1 Also Department of Astronomy, Faculty of Science, University of Tokyo

Dense gas in molecular cloud cores is essential in researches of extragalactic star formation (e.g. Solomon, Downes, and Radford ${ }^{[1]}$ ). To determine the physical relations between star-forming regions and dense gas, we have observed CS $(J=3 \rightarrow 2)$ in starburst galaxies $M 82$ and NGC253. The $J=3$ level of CS is $14.1 \mathrm{~K}$ and the critical density for excitation is $4 \times 10^{5} \mathrm{~cm}^{-3}$.

Maps of the CS $(J=3 \rightarrow 2)$ of NGC253 are illustrated in Fig. 1. The fraction of continuum emission is so small that it has not been subtracted in Fig. 1. The CS $(J=3 \rightarrow 2)$ emission is separated in velocity; the redand blue-velocity components are located west and east of the nucleus, respectively. This is similar to the $\mathrm{HCN}(J=1 \rightarrow 0)$ and $\mathrm{HCO}^{+}(J=1 \rightarrow 0)$ maps by Carlstrom et al. ${ }^{[2]}$.

A map of the $2 \mathrm{~mm}$ continuum and maps of the CS $(J=3 \rightarrow 2)$ of M82 are illustrated in Fig. 2. The continuum has been subtracted at each visibility spectrum before the CS $(J=3 \rightarrow 2)$ maps are made. The CS $(J=3 \rightarrow 2)$ emission is distinctly stronger in the east side of the center than in the west side. This is quite different from any other tracer; centimeter-[s] and millimeter-wave radio continuum (Figure 2 and ref. $[2])$, mid-infrared continuum ${ }^{[4]}, \mathrm{HI}^{[5]}, \mathrm{CO}(J=1 \rightarrow 0)^{[6]}, \mathrm{CO}(J=2 \rightarrow 1)^{[7]}$, HCN $(J=1 \rightarrow 0)^{[2]}$, and $\mathrm{HCO}^{+}(J=1 \rightarrow 0)^{[2]}$ show two peaks with similar strengths (or rather stronger in the west). The upper limit at the west side is consistent with the CS $(J=3 \rightarrow 2)$ spectrum by Mauersberger et al. ${ }^{[8]}$. The peculiar distribution of CS $(J=3 \rightarrow 2)$ in M82 is not explained simply by the difference between the temperatures of the dense gas in both sides of the center since the multi-line study by Wild et al. ${ }^{[9]}$ indicates that the line strengths of higher transitions of $\mathrm{CO}, \mathrm{C}^{18} \mathrm{O}, \mathrm{HCN}$, and $\mathrm{HCO}^{+}$are not weak at the west peak. The underlying mechanism for this difference is not yet understood.

\section{REFERENCES}

[1] Solomon, P.M., Downes, D., and Radford, S.J.E. 1992, ApJL, 387, L55

[2] Carlstrom, J.E. 1988, Ph.D Thesis at U. C. Berkeley

[3] Kronberg, P.P., Biermann, P., and Schwab, F.R. 1985, ApJ, 291, 693

[4] Rieke, G.H. et al. 1980, ApJ, 238, 24

[5] Weliachew, L., Fomalont, E.B., and Greisen, E.W. 1984, A\&A 137, 335

[6] Lo, K.Y. et al. 1987, ApJ, 312, 574

[7] Loiseau, N. et al. 1990, A\&A, 228, 331

[8] Mauersberzer, R. et al. 1991, A\&A, 247, 307

[9] Wild, W. et al. 1992, A\&A, 265, 447 

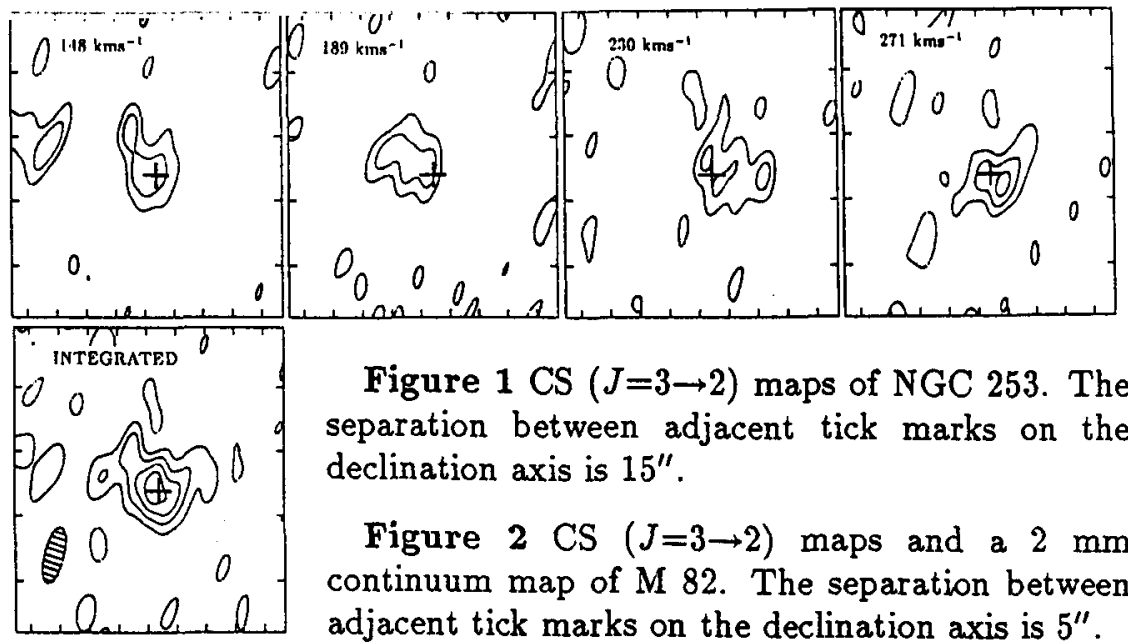

Figure 1 CS $(J=3 \rightarrow 2)$ maps of NGC 253. The separation between adjacent tick marks on the declination axis is $15^{\prime \prime}$.

Figure $2 \mathrm{CS}(J=3 \rightarrow 2)$ maps and a $2 \mathrm{~mm}$ continuum map of $M 82$. The separation between adjacent tick marks on the declination axis is $5^{\prime \prime}$.

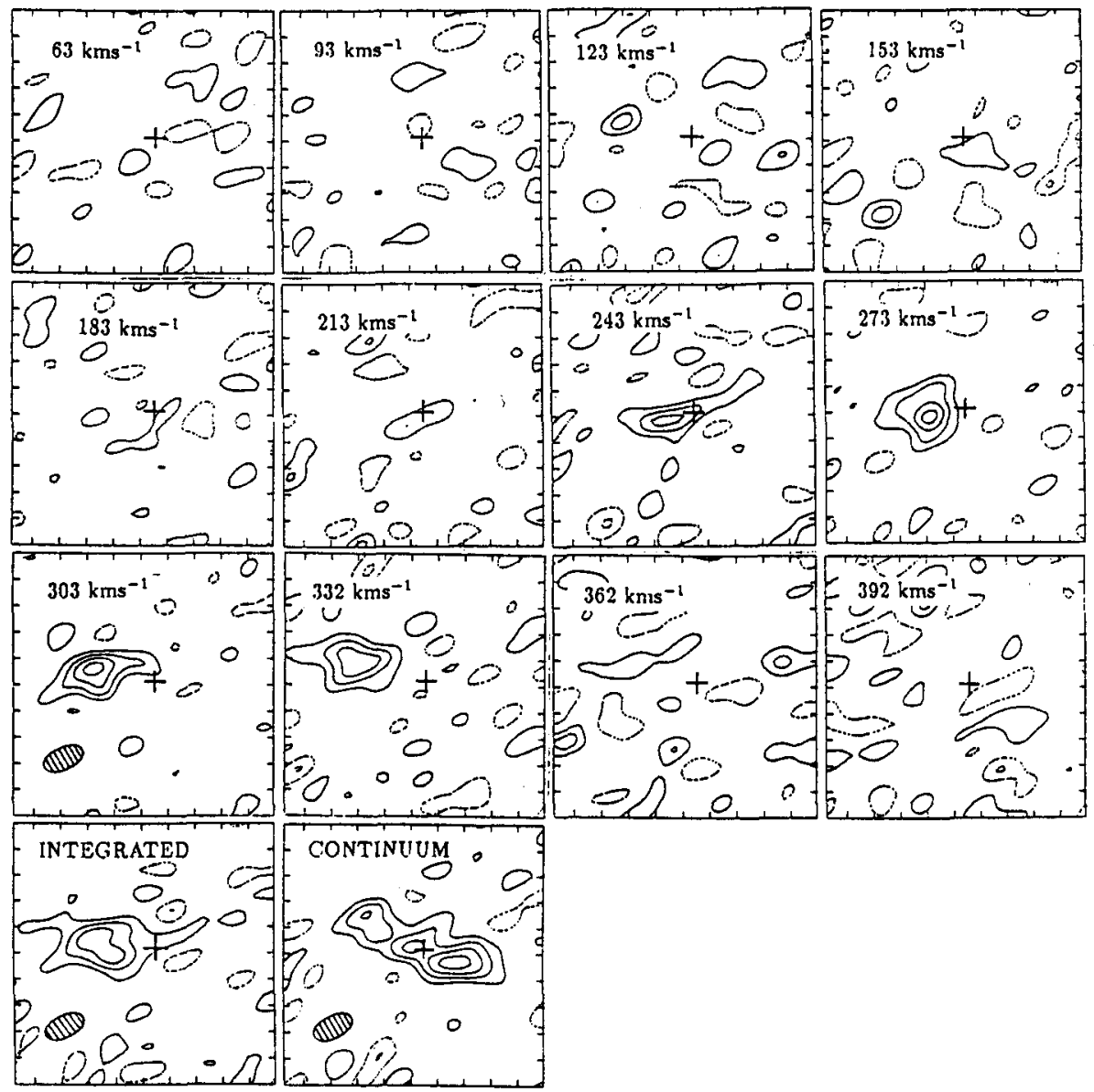

